\title{
ON THE DIFFERENTIABILITY OF A DISTANCE FUNCTION
}

\author{
Kwang-Soon Park
}

\author{
Communicated by Stevan Pilipović
}

\begin{abstract}
Let $M$ be a simply connected complete Kähler manifold and $N$ a closed complete totally geodesic complex submanifold of $M$ such that every minimal geodesic in $N$ is minimal in $M$. Let $U_{\nu}$ be the unit normal bundle of $N$ in $M$. We prove that if a distance function $\rho$ is differentiable at $v \in U_{\nu}$, then $\rho$ is also differentiable at $-v$.
\end{abstract}

\section{Introduction}

Let $N$ be a closed submanifold of a complete Riemannian manifold $M$ and $\pi: U_{\nu} \mapsto N$ the unit normal bundle of $N$ in $M$. For $v \in T_{p} M, p \in M$, throughout this paper, let $\gamma_{v}(t)$ denote always the geodesic curve such that $\gamma_{v}(0)=p$ and $\gamma_{v}^{\prime}(0)=v$. Define a function $\rho: U_{\nu} \mapsto \mathbb{R}$ by

$$
\rho(v):=\sup \left\{t>0 \mid d\left(N, \gamma_{v}(t)\right)=t\right\} \quad \text { for } v \in U_{\nu},
$$

where $d\left(N, \gamma_{v}(t)\right)$ denotes the distance between $N$ and $\gamma_{v}(t)$. For each positive integer $k \in \mathbb{N}$, define a function $\lambda_{k}: U_{\nu} \mapsto \mathbb{R}$ by

$$
\lambda_{k}(v):=\sup \left\{t>0\left|\gamma_{v}\right|_{[0, t]} \text { has no } k \text {-th focal point of } N\right\}
$$

for $v \in U_{\nu}$ [2]. The followings are well known: $\rho$ is continuous [10] and $\lambda_{1}$ is smooth where $\lambda_{1}$ is finite [2]. Itoh and Tanaka [2] proved that the function $\rho$ on $U_{\nu}$ is locally Lipschitz, where $\rho$ is finite. So, by Rademacher's theorem ([1], [6]), the function $\min (\rho, r)$ is differentiable almost everywhere for each $r>0$. Generally, it is well known that $\rho$ is differentiable at $v \in U_{\nu}$ if $\gamma_{v}(\rho(v))$ is a normal cut point, i.e., there exist exactly two $N$-segments through $\gamma_{v}(\rho(v))$ such that $\gamma_{v}(\rho(v))$ is not a focal point along all of these two $N$-segments. Furthermore, in the case $\operatorname{dim} M=2$, Tanaka [8] proved that a point $v \in U_{\nu}$ with $\rho(v)<\infty$ is a differentiable point of the function $\rho$ if and only if $\gamma_{v}(\rho(v))$ is a 1-st focal point of $N$ along $\gamma_{v}$ or there exist at most two $N$-segments through $\gamma_{v}(\rho(v))$. Here, a curve $\gamma:[0, r] \mapsto M$ is called

2000 Mathematics Subject Classification: Primary 53C22; 53C55.

Supported by the BK21 project of the Ministry of Education, Korea. 
an $N$-segment if $\gamma$ is a geodesic curve such that $\gamma^{\prime}(0) \in U_{\nu}$ and $d(N, \gamma(t))=t$ for $t \in[0, r]$. This fact is obviously very nice but didn't have any information about the $n$-dimensional manifold $M$ with $n \geqslant 3$. So, we plan to consider the manifold $M$ such that it has some good conditions. Then we have

MAIN TheOREM. Let $M$ be a simply connected complete Kähler manifold and $N$ a closed complete totally geodesic complex submanifold of $M$ such that every minimal geodesic in $N$ is minimal in $M$. Let $U_{\nu}$ be the unit normal bundle of $N$ in $M$. If $\rho$ is differentiable at $v \in U_{\nu}$, then $\rho$ is also differentiable at $-v$.

\section{Proof of the Main Theorem}

Now, we need the following theorem

Ambrose Theorem. Let $M$ and $\widetilde{M}$ be $m$-dimensional complete Riemannian manifolds and $I: T_{p} M \mapsto T_{\widetilde{p}} \widetilde{M}$ a linear isometry. Suppose that $M$ is simply connected and for any once broken geodesic $\gamma:[0, l] \mapsto M$ in $M$

$$
I_{t}(R(u, v) w)=\widetilde{R}\left(I_{t}(u), I_{t}(v)\right) I_{t}(w)
$$

for any $u, v, w \in T_{\gamma(t)} M, 0 \leqslant t \leqslant l$, where $R$ and $\widetilde{R}$ denote the curvature tensors of $M$ and $\widetilde{M}$, respectively. For any minimal geodesic $\gamma:[0, l] \mapsto M$ with $\gamma(0)=$ $p$, define a geodesic $\widetilde{\gamma}$ by $\widetilde{\gamma}(t):=\gamma_{I\left(\gamma^{\prime}(0)\right)}(t)$ and define a map $\Phi: M \mapsto \widetilde{M}$ by $\Phi(\gamma(t)):=\widetilde{\gamma}(t)$. Then $\Phi$ is well defined and a $C^{\infty}$ Riemannian covering. In particular, if $\widetilde{M}$ is also simply connected, then $M$ and $\widetilde{M}$ are isometric [7].

In our case, since $M$ is complete Kähler, let $g$ and $I$ denote the corresponding Kähler metric and the corresponding complex structure, respectively. Let $\nabla$ and $R$ be the Levi-Civita connection and the curvature tensor of the metric $g$, respectively. For each $p \in M$, we know that $\left.I\right|_{T_{p} M}: T_{p} M \mapsto T_{p} M$ is a linear isometry, where $\left.I\right|_{T_{p} M}$ means the restriction of the complex structure $I$ to the tangent space $T_{p} M$. We see $\nabla I=0$. Furthermore [5], $R(I, I)=R($, ) and $I \circ R=R \circ I$. For any minimal geodesic $\gamma:[0, l] \mapsto M$ with $\gamma(0)=p$, define a map $\Phi_{p}: M \mapsto M$ by

$$
\Phi_{p}(\gamma(t)):=\gamma_{I\left(\gamma^{\prime}(0)\right)}(t) \text { for } t \in[0, l]
$$

Then, by Ambrose Theorem, $\Phi_{p}$ is an isometry for each $p \in M$.

Proposition 1. $\Phi_{p}(N)=N$ for each $p \in N$.

Proof. Firstly, we claim $\Phi_{p}(N) \supset N$. For any $q \in N$, there exists a minimal geodesic curve $\gamma:[0,1] \mapsto N$ such that $\gamma(0)=p$ and $\gamma(1)=q$. By the hypothesis, $\gamma$ is also a minimal geodesic curve in $M$. Since $\Phi_{p}$ is isometric and $N$ is complex, $\Phi_{p}^{k} \circ \gamma$ is minimal in $N$ for each $k \in\{1,2,3,4\}$. Hence,

$$
q=\left(\Phi_{p}^{4} \circ \gamma\right)(1)=\Phi_{p}\left(\Phi_{p}^{3}(\gamma(1))\right) \in \Phi_{p}(N) .
$$

Secondly, we claim $\Phi_{p}(N) \subset N$. For any $q \in \Phi_{p}(N)$, by definition, there exists a point $\widetilde{q} \in N$ such that $\Phi_{p}(\widetilde{q})=q$. Choose a minimal geodesic curve $\gamma:[0,1] \mapsto N$ such that $\gamma(0)=p$ and $\gamma(1)=\widetilde{q}$. Then, $\gamma$ is also minimal in $M$. As the above, $\Phi_{p} \circ \gamma$ is minimal in $N$. Thus, $\left(\Phi_{p} \circ \gamma\right)(1)=q \in N$. This completes the proof. 
Proof of the Main Theorem. Since $(M, I)$ is a complex manifold, there exists an atlas $\left\{\left(z_{\alpha}, U_{\alpha}\right) \mid \alpha \in A\right\}$ of $M$, being a subfamily of the maximal atlas of $M$, such that

(i) $\left\{U_{\alpha} \mid \alpha \in A\right\}$ is a locally finite open covering of $M$,

(ii) there exists a partition of unity $\left\{\varphi_{\alpha}: M \mapsto \mathbb{R} \mid \alpha \in A\right\}$ such that $\operatorname{supp} \varphi_{\alpha} \subset$ $U_{\alpha}$ for all $\alpha \in A$.

Let $\pi: T M \mapsto M$ be the natural projection map, given by $\pi(p, v)=p$ for $(p, v) \in T M$. Conveniently, identify the tangent space $T M$ with the holomorphic tangent space $T^{\prime} M[5]$. Given a chart $z_{\alpha}: U_{\alpha} \mapsto \mathbb{C}^{m}, \alpha \in A$, we can naturally have the corresponding chart $d z_{\alpha}: T^{\prime} U_{\alpha} \mapsto \mathbb{C}^{m} \times \mathbb{C}^{m}$ by

$d z_{\alpha}(v)=\left(z_{\alpha}^{1}, z_{\alpha}^{2}, \ldots, z_{\alpha}^{m} ; \xi_{\alpha}^{1}, \xi_{\alpha}^{2}, \ldots, \xi_{\alpha}^{m}\right)$, where $v=\sum_{k=1}^{m} \xi_{\alpha}^{k} \frac{\partial}{\partial z_{\alpha}^{k}} \in T_{p}^{\prime} U_{\alpha}$ with $p \in U_{\alpha}$.

For $v^{\prime}, w^{\prime} \in T_{v}^{\prime}(T M)$ with $v \in T U_{\alpha}\left(\equiv T^{\prime} U_{\alpha}\right)$ and $\alpha \in A$ let their coordinate representations be $\left(v_{\alpha 1}^{\prime}, \ldots, v_{\alpha m}^{\prime} ; \eta_{\alpha 1}, \ldots, \eta_{\alpha m}\right)$ and $\left(w_{\alpha 1}^{\prime}, \ldots, w_{\alpha m}^{\prime} ; \eta_{\alpha 1}^{\prime}, \cdots, \eta_{\alpha m}^{\prime}\right)$. Then we put

$$
h\left(v^{\prime}, w^{\prime}\right):=\sum_{\substack{\alpha \in A \\ v \in T U_{\alpha} \\ i \in\{1, \ldots, m\}}} \varphi_{\alpha}(p)\left(v_{\alpha i}^{\prime} \overline{w_{\alpha i}^{\prime}}+\eta_{\alpha i} \overline{\eta_{\alpha i}^{\prime}}\right),
$$

where $p=\pi(v)$. This defines a Hermitian metric on the complex manifold $T M$. Let $G$ be the Riemannian metric on $T M$ which is naturally induced from the Hermitian metric $h$.

Assume that $\rho$ is differentiable at $v \in U_{\nu} \cap T_{p} M$. By definition, the differential $d \Phi_{p}$ of the map $\Phi_{p}$ has the following properties

$$
\left(d \Phi_{p}\right)_{p}(v)=I v \quad \text { and } \quad\left(d \Phi_{p}\right)_{p}(I v)=I(I v)=-v .
$$

We know that $\rho$ is differentiable at $v \in U_{\nu}$ if and only if for any unit speed smooth curve $c:(-\epsilon, \epsilon) \mapsto U_{\nu}$ with $c(0)=v$ and $\epsilon>0$ the following limit exists:

$$
\lim _{t \rightarrow 0} \frac{\rho(c(t))-\rho(c(0))}{t} .
$$

Take any unit speed smooth curve $\widetilde{c}:(-\epsilon, \epsilon) \mapsto U_{\nu}$ with $\widetilde{c}(0)=I v$ and sufficiently small $\epsilon>0$. Let $p_{t}:=\pi(-I \widetilde{c}(t))$ for each $t \in(-\epsilon, \epsilon)$. By Proposition 1 ,

$$
d\left(N, \gamma_{\widetilde{c}(t)}(s)\right)=d\left(\Phi_{p_{t}}(N), \Phi_{p_{t}}\left(\gamma_{-I \widetilde{c}(t)}(s)\right)\right)=d\left(N, \gamma_{-I \widetilde{c}(t)}(s)\right)
$$

for all $s \in\left[0, l_{t}\right]$ with $l_{t}:=\sup \left\{r>0\left|\gamma_{-I \widetilde{c}(t)}\right|_{[0, r]}\right.$ is minimal $\}$ so that

$$
\rho(\widetilde{c}(t))=\sup \left\{s>0 \mid d\left(N, \gamma_{-I \widetilde{c}(t)}(s)\right)=s\right\}=\rho(-I \widetilde{c}(t))
$$

for $t \in(-\epsilon, \epsilon)$. Note that $-I \widetilde{c}(t)$ is a unit speed smooth curve in $U_{\nu}$ with the property $-I \widetilde{c}(0)=v$. Thus, by the hypothesis, the following limit

$$
\lim _{t \rightarrow 0} \frac{\rho(\widetilde{c}(t))-\rho(\widetilde{c}(0))}{t}=\lim _{t \rightarrow 0} \frac{\rho(-I \widetilde{c}(t))-\rho(-I \widetilde{c}(0))}{t}
$$

exists. Hence, $\rho$ is differentiable at $I v$. Furthermore, from this result, $\rho$ is also differentiable at $I(I v)=-v$. Therefore, we complete the proof. 
Remarks. 1. In particular, if $\rho$ is differentiable at $v \in U_{\nu}$, then $\rho$ is also differentiable at $w \in\left\{v, I v, I^{2} v=-v, I^{3} v=-I v\right\}$.

2. Let $\left\langle\Phi_{p}\right\rangle$ be the group generated by the element $\Phi_{p}$. Then $\left\langle\Phi_{p}\right\rangle$ is a cyclic group of order 4. Let $G:=\bigcup_{p \in M}\left\langle\Phi_{p}\right\rangle$. Then $G \subset \operatorname{iso}(M)$, where iso $(M)$ denotes the group of all isometries of $M$

3. For each $p \in M$, let $N=\{p\}$ as a 0 -dimensional complex submanifold of $M$. Then $U_{\nu}=U_{p} M$, where $U_{p} M$ denotes the unit tangent vector space of $M$ at $p$. If $\rho$ is differentiable at $v \in U_{\nu}$, then $\rho$ is also differentiable at $w \in\{v, I v,-v,-I v\}$.

4. Consider the complex projective space $\mathbb{P}^{n}$ with the Fubini-Study metric [3]. Let $\mathbb{P}^{k}:=\left\{\left(z_{0}: \cdots: z_{k}: 0: \cdots: 0\right) \mid z_{i} \in \mathbb{C}, 0 \leqslant i \leqslant k\right\} \subset \mathbb{P}^{n}$ for $k=1, \ldots, n-1$. Then $\mathbb{P}^{n}$ is a simply connected complete Kähler amnifold and $\mathbb{P}^{k}$ is a closed complete totally geodesic complex submanifold of $\mathbb{P}^{n}$ such that every minimal geodesic in $\mathbb{P}^{k}$ is minimal in $\mathbb{P}^{n}$ [4]. Let $U_{\nu}$ be the unit normal bundle of $\mathbb{P}^{k}$ in $\mathbb{P}^{n}$. If $\rho$ is differentiable at $v \in U_{\nu}$, then $\rho$ is also differentiable at $w \in\{v, I v,-v,-I v\}$.

5. Let $(M, g)$ be a simply connected complete Riemannian manifold with a hyperkähler structure $(g, I, J, K)$ and $N$ a closed complete totally geodesic trianalytic submanifold of $M$ such that every minimal geodesic in $N$ is minimal in $M$ ([3], [9]). If $\rho$ is differentiable at $v \in U_{\nu}$, then $\rho$ is also differentiable at $w \in\left\{R^{i} v \mid i \in\{1,2,3,4\}, R \in S^{2}\right\}$, where $S^{2}:=\left\{a I+b J+c K \mid a^{2}+b^{2}+c^{2}=1\right\}$.

Now, we consider

Question 1. Let $M$ be a simply connected complete Kähler manifold and $N$ a closed complete totally geodesic complex submanifold of $M$. Then, is it true that every minimal geodesic in $N$ is also minimal in $M$ ?

The author believes that it may be true, but can not prove it.

QUestion 2. Let $(M, g, I)$ be a 2-dimensional simply connected complete Kähler manifold and $N$ a 1-dimensional closed complex submanifold of $M$. Let $U_{\nu}$ be the unit normal bundle of $N$ in $M$. Then, at which $v \in U_{\nu}$ is $\rho: U_{\nu} \mapsto \mathbb{R}$ differentiable?

Note that if $v \in T_{p} N$ with $g(v, v)=1$ and $u \in T_{p} M \cap U_{\nu}$ for $p \in N$, then we easily get

$$
T_{p} M=\mathbb{R}\langle v, I v, u, I u\rangle \quad \text { and } \quad T_{p} M \cap U_{\nu}=\left\{a u+b I u \mid a^{2}+b^{2}=1\right\} .
$$

\section{References}

[1] H. Federer, Geometric Measure Theory, Springer-Verlag, Berlin-Heidelberg-New York, 1969.

[2] J. Itoh and M. Tanaka, The Lipschitz continuity of the distance function to the cut locus, Trans. Amer. Math. Soc. 353 (2001), 21-40.

[3] D. D. Joyce, Compact Manifolds With Special Holonomy, Oxford University Press, 2000.

[4] Y.H. Kim and S. Maeda, A practical criterion for some submanifolds to be totally geodesic, Monatsh. Math. 149 (2006), 233-242.

[5] S. Kobayashi and K. Nomizu, Foundations of Differential Geometry, John Wiley and Sons, 1969.

[6] F. Morgan, Geometric Measure Theory, A Beginner's Guide, Academic Press, 1988.

[7] T. Sakai, Riemannian Geometry, Translation of Mathematical Monographs, vol. 149, Amer. Math. Soc., 1996. 
[8] M. Tanaka, Characterization of a differentiable point of the distance function to the cut locus, J. Math. Soc. Japan 55 (2003), 231-241.

[9] M. Verbitsky and D. Kaledin, Hyperkähler Manifolds, Internatioanal Press, 1999.

[10] J.H.C. Whitehead, On the covering of a complete space by the geodesics through a point, Ann. of Math. 36 (1935), 679-704.

School of Mathematical Sciences

(Received 2703 2007)

Seoul National University

(Revised 1501 2008)

Seoul 151-747

Korea

parkksn@math.snu.ac.kr 